ВІСНИК

ОДЕСЬКОГО НАЦІОНАЛЬНОГО

МОРСЬКОГО УНІВЕРСИТЕТУ

№ 2 (65), 2021
HERALD

OF THE ODESSA NATIONAL

MARITIME UNIVERSITY № 2 (65), 2021

УДК 620:178.3

DOI 10.47049/2226-1893-2021-2-128-137

\title{
СРАВНИТЕЛЬНАЯ ОЦЕНКА ЭКСПЕРИМЕНТАЛЬНЫХ И РАСЧЁТНЫХ ЗНАЧЕНИЙ РАЗРУШАЮЩИХ НАПРЯЖЕНИЙ ПРИ ИСПЫТАНИЯХ С ЛИНЕЙНО ВОЗРАСТАЮЩЕЙ НАГРУЗКОЙ
}

\author{
А.В. Коноплёв \\ д.т.н., профессор, заведующий кафедрой «Машиноведение» \\ А.Н. Шумило \\ к.т.н., профессор, доцент кафедры «Машиноведение» \\ Е.К. Рожко \\ ассистент кафедры «Прикладная механика» \\ В.В. Галевский, О.Н. Кононова \\ старшие преподаватели кафедры «Машиноведение»
}

Одесский национальный морской университет, Одесса, Украина

Аннотация. В статье проведена сравнительная оценка экспериментальных и расчётных значений разрушающих напряжений при линейно возрастающей скорости увеличения нагрузки.

Для этого были использованы корреляциионные зависимости между пределом выносливости и параметрами наклонного участка кривой усталости, выраженной степенным уравнением и уравнением Вейбулла. Показано, что минимальное расхождение между экспериментальными и расчётными значениями разрушающих напряжений имеет место в случае использования корреляционных зависимостей между параметрами уравнения Вейбулла. Полученные результать дают возможность расчётным путем прогнозировать систематическую погрешность ускоренных методов определения усталостной прочности объектов, в которых используется принции плавного нагружения при линейно возрастающей нагрузке.

Ключевые слова: ускоренные испытания, разрушающее напряжение, скорость увеличения нагрузки.

УДК 620:178.3

DOI 10.47049/2226-1893-2021-2-128-137

\section{ПОРІВНЯЛЬНА ОЩІНКА ЕКСПЕРИМЕНТАЛЬНИХ І РОЗРАХУНКОВИХ ЗНАЧЕНЬ РУЙНІВНИХ НАПРУЖЕНЬ ПРИ ВИПРОБУВАННЯХ З ЛІНІЙНО ЗРОСТАЮЧИМ НАВАНТАЖЕННЯМ}

А.В. Конопльов

д.т.н., професор, завідувач кафедри «Машинознавство» О.М. Шумило к.т.н., професор, доцент кафедри «Машинознавство»

О.К. Рожко асистент кафедри «Прикладна механіка»

В.В. Галевський, О.М. Кононова

старші викладачі кафедри «Машинознавство»

Одеський національний морський університет

(С Коноплев А.В., Шумило О.М., Рожко О.К., Галевський В.В., Кононова О.М., 2021 
Анотація. У статті проведена порівняльна оцінка експериментальних і розрахункових значень руйнівних напружень при лінійно зростаючій швидкості збільшення навантаження.

Для ц̧ього були використані корелячуійні залежності між межею витривалості і параметрами похилої ділянки кривої втоми, вираженоі степеневим рівнянням і рівнянням Вейбулла. Показано, щуо мінімальне розходження між експериментальними і розрахунковими значеннями руйнівних напружень має місце в разі використання кореляційних залежностей між параметрами рівняння Вейбулла. Отримані результати дають можливість розрахунковим шляхом прогнозувати систематичну похибку прискорених методів визначення втомної міцності об'єктів, в яких використовується принцип плавного навантаження при лінійно зростаючому навантаженні.

Ключові слова: прискорені випробування, руйнівне напруження, швидкість збільшення навантаження.

UDC 620:178.3

DOI 10.47049/2226-1893-2021-2-128-137

\title{
COMPARATIVE ASSESSMENT OF EXPERIMENTAL AND CALCULATED VALUES OF BREAKING TENSIONS DURING TESTS WITH A LINEARLY INCREASING LOAD
}

\author{
A.V. Konoplev \\ Doctor of Technical Sciences, Professor, \\ Head of the Department of «Machine Science» \\ A.N. Shumilo \\ Ph.D Associate Professor of the Department of «Machine Science» \\ E.K. Rozhko \\ Assistant of the Department «Applied mechanics» \\ V.V. Halevskyi, O.N. Kononova \\ Senior Lecturers of the Department «Machine Science» \\ Odessa National Maritime University, Odessa, Ukraine
}

\begin{abstract}
The article provides a comparative assessment of the experimental and calculated values of the breaking tensions at a linearly increasing rate of increase in the load.

For this, the correlations between the endurance limit and the parameters of the inclined portion of the fatigue curve expressed by the power equation and the Weibull equation were used. It is shown that the minimum discrepancy between the experimental and calculated values of the breaking tensions occurs in the case of using the correlation dependences between the parameters of the Weibull equation. The results obtained make it possible by calculation to predict the systematic error of accelerated methods for determining the fatigue strength of objects, in which the principle of smooth loading with a linearly increasing load is used.
\end{abstract}

Keywords: accelerated testing, breaking tension, load increase rate. 
Введение. Вопросы материалоёмкости и качества всегда находились в центре внимания предприятий, занимающихся производством и ремонтом изделий машиностроения. Высокое качество продукции и оптимальная материалоёмкость достигаются тщательной отработкой каждой детали, а, следовательно, и изделия в целом на всех этапах её создания, модернизации или ремонта.

Применение современных методов расчёта несущей способности деталей и элементов конструкций позволяют обеспечить минимальную их материалоёмкость. Тем не менее, в ряде случаев, например, для проверки принятого конструктивного решения или при контроле качества серийно выпускаемой продукции часто возникает необходимость в проведении натурных испытаний на сопротивление усталости деталей или их отдельных элементов. Натурные испытания могут также понадобиться в случае контроля процесса восстановления деталей различными способами (наплавкой, напылением, гальваническими покрытиями и др.). Такие испытания актуальны и при замене одного материала другим, близким ему по свойствам.

Испытание деталей на сопротивление усталости обычными методами для получения соответствующих характеристик с требуемой степенью точности требует большого количества объектов испытаний и большой продолжительности эксперимента, т.е. связано с большими материальными затратами. Особенно это касается испытаний по определению предела выносливости на большой базе. Принимая во внимание сказанное, напрашивается вывод о целесообразности замены длительных испытаний ускоренными, которые при сохранении точности позволили бы существенно сократить время испытаний и количество испытуемых объектов. Запрос на разработку ускоренных методов привёл к формированию отдельного направления, объединившего различные подходы к решению данной проблемы. В частности в последние годы в рамках этого направления были исследованы вопросы разработки новых и совершенствования существующих ускоренных методов, их точности и классификации [1;2.].

Настоящая статья посвящена одному из этих вопросов, в частности, вопросу оценки точности ускоренных методов, в которых используется принцип плавного увеличения нагрузки в процессе проведения эксперимента. Согласно упомянутой выше классификации ускоренные методы, основанные на таком подходе, объединены общим режимным признаком.

Целью статьи является проверка соответствия расчётных и экспериментальных разрушающих напряжений, соответствующих режиму плавного линейного увеличения нагрузки.

Изложение основного материала. Для расчётного определения разрушающих напряжений примем три исходные предпосылки. Вопервых, будем полагать, что при увеличении амплитуды напряжений суммирование усталостных повреждений подчинено линейной гипотезе. 
ВІСНИК

ОДЕСЬКОГО НАЦІОНАЛЬНОГО

МОРСЬКОГО УНІВЕРСИТЕТУ

№ 2 (65), 2021
HERALD

OF THE ODESSA NATIONAL

MARITIME UNIVERSITY № 2 (65), 2021

Во-вторых, в качестве модели кривой усталости примем для сравнения два уравнения - степенное и Вейбулла. В-третьих, параметры этих уравнений будем определять на основе их корреляционных зависимостей от предела выносливости. Схема линейного нагружения объекта (образца, детали, модели, отдельного элемента и др.) с постоянной скоростью увеличения амплитуды напряжений $\alpha=d \sigma / d N$ представлена на рис. 1.

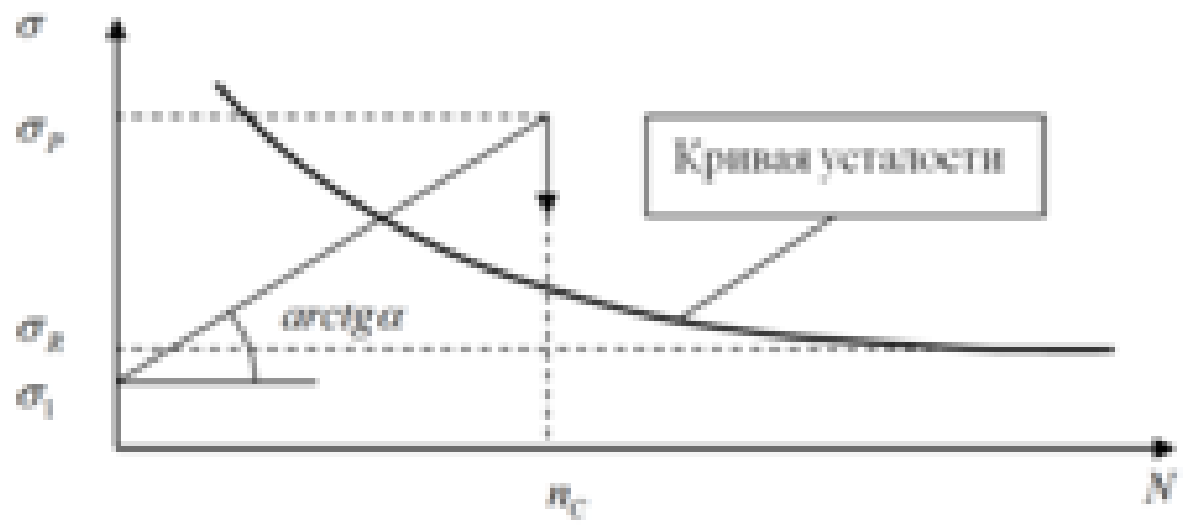

Рис. 1. Схема испытаний объектов при линейно увеличивающейся амплитуде напряжений

На рисунке приняты следующие обозначения:

$\sigma_{R}-$ предел выносливости;

$\sigma$ и $N$ - текущие значения напряжений и числа циклов соответственно;

$\sigma_{1}$ - начальное напряжение;

$n_{C}$ - суммарное число циклов до окончательного разрушения, регистрируемое счётчиком числа оборотов;

$\sigma_{P}-$ напряжение, при котором произошло разрушение, которое, как видно из рис. 1, можно определить по формуле

$$
\sigma_{P}=\sigma_{1}+\alpha n_{C}
$$

Параметры наклонных участков кривих усталости степенного уравнения $m_{C}$ и $C_{C}$, а также уравнения Вейбулла $m_{W}$ и $C_{W}$ будем определять с помощью известных выражений [3; 4]

$$
m_{C}=f_{C}\left(\sigma_{R}\right)=a_{C} \sigma_{R}+b_{C}
$$




$$
\begin{gathered}
C_{C}=F_{C}\left(\sigma_{R}\right)=\alpha_{C}\left(m_{C}+1\right) \lg \sigma_{R}+\beta_{C} ; \\
m_{W}=f_{W}\left(\sigma_{R}\right)=\frac{1}{a_{W 1} \sigma_{R}^{0,01}-b_{W 1}}-1=\frac{1}{5,254 \sigma_{R}^{0,01}-5,038}-1 ; \\
C_{W}=F_{W}\left(\sigma_{R}\right)=\lg \frac{\left(\alpha_{W 1} \sigma_{R}+\beta_{W 1}\right)^{m_{W}+1}}{m_{W}+1}=\lg \frac{\left(7,029 \sigma_{R}+3599\right)^{m_{W}+1}}{m_{W}+1},
\end{gathered}
$$

где $a_{C}, b_{C}, \alpha_{C}, \beta_{C}, a_{W 1}, b_{W 1}, \alpha_{W 1}, \beta_{W 1}$ - эмпирические коэффициенты. Для объектов из углеродистых и легированных сталей принимают: $a_{C}=0,027 ; \quad b_{C}=1,4 ; \quad \alpha_{C}=0,997 ; \quad \beta_{C}=4,0+0,5\left(2,5-\lg \sigma_{R}\right)^{2}, \quad$ при $\lg \sigma_{R} \geq 2,5 \beta_{C}=4,0$ (в среднем $\beta_{C}=4,25$ ).

Расчётные величины разрушающих напряжений при выбранных значениях скорости увеличения нагрузки $\alpha$ будем определять по следующим формулам

$$
\begin{gathered}
\sigma_{P}^{C}=\left[\sigma_{R}^{m_{c}+1}+\alpha\left(m_{c}+1\right) 10^{C_{c}}\right]^{1 /\left(m_{c}+1\right)} ; \\
\sigma_{P}^{W}=\sigma_{R}+\left[\alpha\left(m_{W}+1\right) 10^{C_{W}}\right]^{1 /\left(m_{W}+1\right)} .
\end{gathered}
$$

Для сравнения расчётных и экспериментальных значений разрушающих напряжений воспользуемся приведенными в литературе [5] результатами испытаний гладких и надрезанных лабораторных образцов при круговом изгибе, изготовленных из двух марок конструкционной стали (45 и 40X). Предварительно для них определим параметры $m_{C}, C_{C}$, $m_{W}$ и $C_{W}$. Результаты расчёта приведены в табл. 1.

В таблице приняты обозначения: образцы I типа - гладкие, а II типа - надрезанные. Результаты расчёта величин разрушающих напряжений $\sigma_{P}^{C}$ и $\sigma_{P}^{W}$ по формулам (6) и (7), а также средние значения $\bar{\sigma}_{P}^{\ni}$, рассчитанные по экспериментальным данным, приведены в табл. 2. В этой же таблице приведены отклонения расчётных и экспериментальных разрушающих напряжений для двух сравниваемых уравнений кривой усталости $\delta_{\sigma_{P}^{C}}, \delta_{\sigma_{P}^{W}}$, которые были определены по следующим формулам:

$$
\begin{aligned}
& \delta_{\sigma_{P}^{C}}=\frac{\sigma_{P}^{C}-\sigma_{P}^{\ni}}{\sigma_{P}^{\ni}} \cdot 100 ; \\
& \delta_{\sigma_{P}^{W}}=\frac{\sigma_{P}^{W}-\sigma_{P}^{\ni}}{\sigma_{P}^{\ni}} \cdot 100 .
\end{aligned}
$$


ВІСНИК

ОДЕСЬКОГО НАЦІОНАЛЬНОГО

МОРСЬКОГО УНІВЕРСИТЕТУ

№ 2 (65), 2021
HERALD

OF THE ODESSA NATIONAL

MARITIME UNIVERSITY

№ 2 (65), 2021

Таблиия 1

Результаты расчёта параметров кривых усталости для гладких и надрезанных образиов при круговом изгибе

\begin{tabular}{|c|c|c|c|c|c|c|}
\hline $\begin{array}{c}\text { Тип } \\
\text { образца }\end{array}$ & Материал & $\begin{array}{c}\sigma_{R}, \\
\text { МПа }\end{array}$ & $m_{C}$ & $C_{C}$ & $m_{W}$ & $C_{W}$ \\
\hline I & Сталь 45 & 250 & 18,1818 & 50,0364 & 0,9446 & 6,9622 \\
\hline II & Сталь 45 & 145 & 6,6667 & 21,0067 & 1,0657 & 7,2547 \\
\hline I & Сталь 40X & 274 & 18,8679 & 52,3962 & 0,9255 & 6,9213 \\
\hline II & Сталь 40X & 159 & 7,7519 & 23,1550 & 1,0442 & 7,1992 \\
\hline
\end{tabular}

Средние расчётные значения $\bar{\sigma}_{P}$ определяли с целью оценки их близости к экспериментальным данным по зависимости

$$
\bar{\sigma}_{P}=\frac{\sigma_{P}^{C}+\sigma_{P}^{W}}{2}
$$

При этом отклонения $\bar{\delta}_{\sigma_{P}}$ рассчитывали по формуле

$$
\bar{\delta}_{\sigma_{P}}=\frac{\bar{\sigma}_{P}-\sigma_{P}^{\ni}}{\sigma_{P}^{\ni}} \cdot 100 .
$$

Модульные средние значения $\bar{\delta}_{\sigma_{P}}^{C}$ и $\bar{\delta}_{\sigma_{P}}^{W}$ вычисляли по абсолютным величинам $\left|\delta_{\sigma_{D}}^{\tilde{N}}\right|$ и $\left|\delta_{\sigma_{D}}^{\mathrm{W}}\right|$ с помощью формул

$$
\begin{aligned}
& \bar{\delta}_{P}^{C}=\frac{\sum_{i=1}^{n}\left|\delta_{P}^{C}\right|}{n} ; \\
& \delta_{P}^{W}=\frac{\sum_{i=1}^{n}\left|\delta_{P}^{W}\right|}{n},
\end{aligned}
$$

где $\quad n$ - общее количество табличных значений $\bar{\delta}_{\sigma_{P}}^{C}$ и $\bar{\delta}_{\sigma_{P}}^{W}(n=12)$.

Зависимости величин $\delta_{\sigma_{P}^{C}}, \delta_{\sigma_{P}^{W}}$ и $\bar{\delta}_{\sigma_{P}}$ от скорости увеличения амплитуды напряжений $\alpha$ представлены на рисунках 1-3. 
ВІСНИК

ОДЕСЬКОГО НАЦІОНАЛЬНОГО

МОРСЬКОГО УНІВЕРСИТЕТУ
HERALD

OF THE ODESSA NATIONAL

MARITIME UNIVERSITY № 2 (65), 2021

Таблица 2

Результаты расчёта величин $\bar{\sigma}_{P}^{\ni}, \sigma_{P}^{C}, \sigma_{P}^{W}, \delta_{\sigma_{P}^{C}}, \delta_{\sigma_{P}^{W}}, \bar{\sigma}_{P} u \bar{\delta}_{\sigma_{P}}$

\begin{tabular}{|c|c|c|c|c|c|c|c|c|c|}
\hline $\begin{array}{l}\text { Тип } \\
\text { обр. }\end{array}$ & Материал & $\begin{array}{c}\alpha, \\
\text { Па/цикл }\end{array}$ & $\begin{array}{l}\bar{\sigma}_{P}^{\ni} \\
\text { МПа }\end{array}$ & $\begin{array}{l}\sigma_{P}^{C} \\
\text { МПа }\end{array}$ & $\begin{array}{l}\sigma_{P}^{W} \\
\text { МПа }\end{array}$ & $\begin{array}{c}\delta_{\sigma_{P}^{C}} \\
\%\end{array}$ & $\begin{array}{c}\delta_{\sigma_{P}^{W}}, \\
\%\end{array}$ & $\begin{array}{c}\bar{\sigma}_{P} \\
\text { МПа }\end{array}$ & $\bar{\delta}_{\sigma_{P}}$, \\
\hline \multirow{2}{*}{ I } & \multirow{6}{*}{ Сталь 45} & 100 & 299,2 & 293,7 & 297,0 & $-1,9$ & $-0,8$ & 295,3 & $-1,3$ \\
\hline & & 200 & 305,7 & 304,2 & 317,1 & $-0,5$ & 3,6 & 310,6 & 1,6 \\
\hline \multirow{4}{*}{ II } & & 100 & 206,3 & 216,9 & 198,5 & 4,9 & $-3,9$ & 207,7 & 0,7 \\
\hline & & 200 & 226,8 & 236,7 & 219,8 & 4,2 & $-3,2$ & 228,3 & 0,6 \\
\hline & & 400 & 245,5 & 258,7 & 249,6 & 5,1 & 1,6 & 254,2 & 3,5 \\
\hline & & 600 & 262,2 & 272,6 & 272,3 & 3,8 & 3,7 & 272,5 & 3,9 \\
\hline \multirow{3}{*}{ I } & \multirow{6}{*}{ Сталь 40X } & 50 & 323,8 & 307,9 & 306,3 & $-5,2$ & $-5,7$ & 307,1 & $-5,2$ \\
\hline & & 100 & 343,9 & 318,0 & 342,9 & $-8,1$ & $-0,3$ & 330,4 & $-3,9$ \\
\hline & & 200 & 353,1 & 328,8 & 340,3 & $-7,4$ & $-3,8$ & 334,5 & $-5,2$ \\
\hline \multirow{3}{*}{ II } & & 50 & 209,3 & 188,3 & 196,1 & $-11,2$ & $-6,7$ & 192,2 & $-8,2$ \\
\hline & & 100 & 217,9 & 201,0 & 211,1 & $-8,4$ & $-3,2$ & 206,0 & $-5,4$ \\
\hline & & 200 & 238,9 & 215,9 & 232,1 & $-10,6$ & $-2,9$ & 224,0 & $-6,2$ \\
\hline \multicolumn{5}{|c|}{ Средние значения } & & $-2,9$ & $-1,8$ & & $-2,1$ \\
\hline \multicolumn{5}{|c|}{ Модульные средние значения $\bar{\delta}_{\sigma_{P}}^{C}$ и $\bar{\delta}_{\sigma_{P}}^{W}$} & & 5,9 & 3,3 & & 3,8 \\
\hline
\end{tabular}

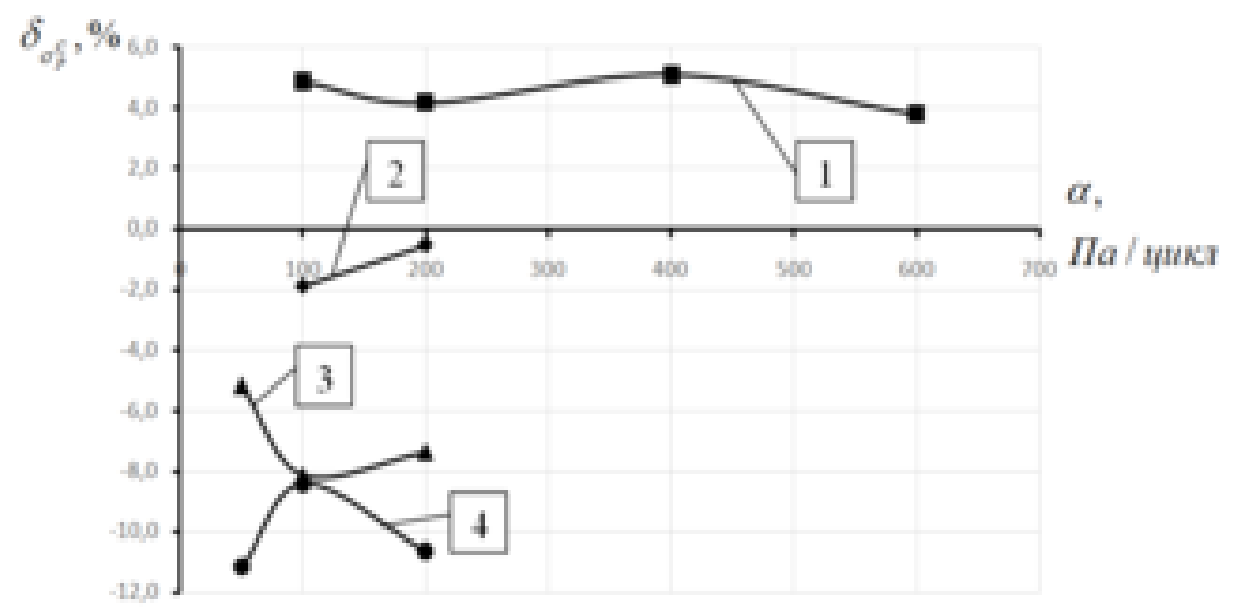

Рис. 1. Зависимость отклонений $\delta_{\sigma_{P}^{C}}$ от скорости увеличения напряжений $\alpha$ для степенного уравнения кривой усталости: 1 - сталь 45 образиы I типа; 2 - сталь 45 образиы IV типа; 3 - сталь 40Х образиы I типа; 4 - сталь 40Х образцы IV типа 
ВІСНИК

ОДЕСЬКОГО НАЦІОНАЛЬНОГО

МОРСЬКОГО УНІВЕРСИТЕТУ
HERALD

OF THE ODESSA NATIONAL

MARITIME UNIVERSITY № 2 (65), 2021

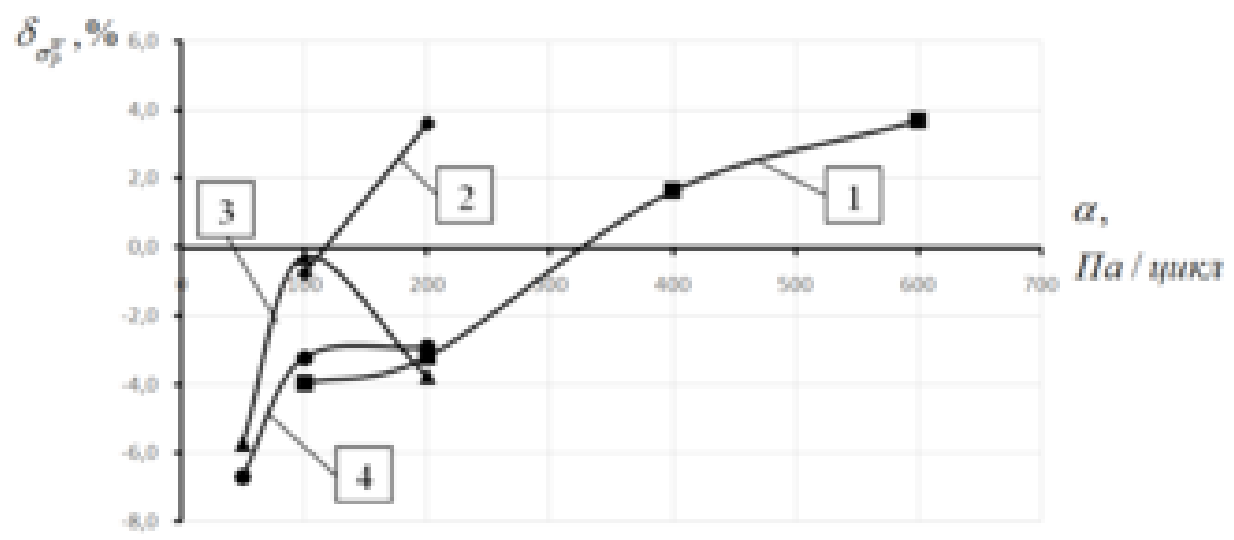

Рис. 2. Зависимость отклонений $\delta_{\sigma_{P}^{W}}$ от скорости увеличения напряжений $\alpha$ для уравнения кривой усталости Вейбулла: 1 - сталь 45 образиы I типа; 2 - сталь 45 образиь IV типа; 3 - сталь 40Х образиы I типа; 4 - сталь 40Х образиы IV типа

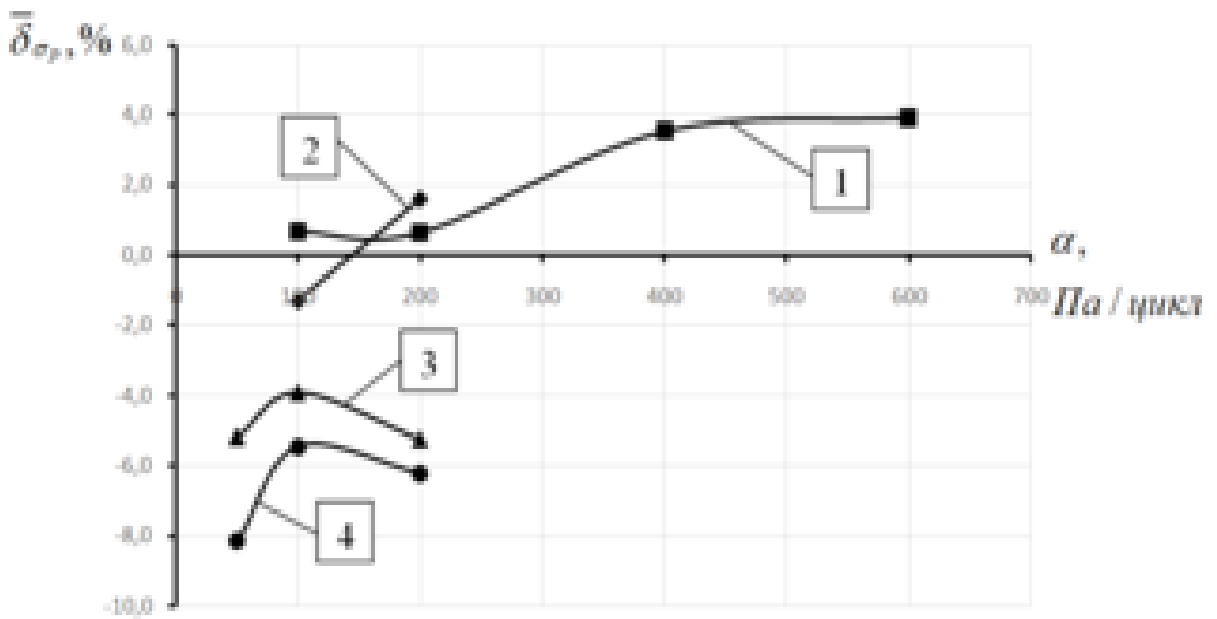

Рис. 3. Зависимость осреднённых отклонений $\bar{\delta}_{\sigma_{P}}$ от скорости $\alpha$ :

1 - сталь 45 образиы I типа; 2 - сталь 45 образиь IV типа; 3 - сталь 40Х образиь I типа; 4 - сталь 40Х образиь IV типа 


\section{Выводы}

1. Выбор тех или иных корреляционных зависимостей между параметрами кривой усталости влияет на различие между расчётными и экспериментальными величинами разрушающих напряжений, а, следовательно, и на точность ускоренных методов, основанных на испытаниях с возрастающей нагрузкой.

2. Проведенные на примере двух уравнений кривой усталости исследования показали, что отклонения расчётных и экспериментальных значений разрушающих напряжений находятся в допустимых пределах. Так максимальные отклонения между ними составили -10,6 \% (для степенного уравнения) и - 6,7 \% (для уравнения Вейбулла). При этом их средние отклонения составили $-2,9 \%$ и $-1,8 \%$, а модульные средние соответственно $5,9 \%$ и $3,3 \%$. Осреднённые значения $\bar{\delta}_{\sigma_{P} \text { оказались }}$ промежуточными и составили соответственно $-2,1 \%$ и 3,8 \%.

3. Скорость увеличения нагрузки, как видно из рисунков 2 и 3 , в основном приводит к росту отклонений между расчётными и экспериментальными величинами разрушающих напряжений.

\section{ЛІТЕРАТУРА}

1. Олейник Н.В., Скляр С.П. Ускоренные испытания на усталость. - К.: Наук. думка, 1985. - 304 c.

2. Коноплёв А.В., Экспериментально-расчётные методы определения предела выносливости деталей машин. Создание их единой классификачии: Автореф. дис. ... докт. техн. наук. Киев, 2013. - 40 с.

3. Олейник Н.В. Выносливость деталей машин. - К.: Техніка, 1979. - 200 c.

4. Коноплёв А.В., Селюкова Е.В. Определение предела выносливости деталей машин методом Про с учётом взаимосвязи между его параметрами // Детали машин: Респ. межвед. науч.-техн. сб. - 1988. - Bып. 40. - C. 102-104.

5. Олейник Н.В., Коноплёв А.В., Кибаков А.Г. Методы ускоренного определения характеристик сопротивления усталости в практических приложениях. - Одесса: Астропринт, 2000. $138 \mathrm{c}$.

\section{REFERENCES}

1. Oleinik N.V., Sklyar S.P. Uskorennie ispitaniya na ustalost' [Accelerated Fatigue Tests]. Kiev: Naukova dumka (Scientific thought), 1985. - 304 l. [in Russian]. 
ВІСНИК

ОДЕСЬКОГО НАЦІОНАЛЬНОГО

МОРСЬКОГО УНІВЕРСИТЕТУ

№ 2 (65), 2021
HERALD

OF THE ODESSA NATIONAL

MARITIME UNIVERSITY № 2 (65), 2021

2. Konoplev A.V. Eksperimentalno-raschetnie metodi opredeleniya predela vinoslivosti detalei mashin. Sozdanie ih edinoi klassifikacii [Experimental and computational methods for determining the endurance limit of machine parts. Creation of their unified classification]: Avtoref. dis...dokt. teh. nauk. (Abstract of the dissertation of doctor of technical sciences) - Kiev, 2013. - $308 \mathrm{l}$.

3. Oleinik N.V., Vinoslivost' detalei mashin [Endurance machine details]. - Kiev: Tehnika (Kiyiv: Technics). 1979. - 200l.

4. Konoplev A.V., Selyukova E.V. Opredelenie predela vinoslivosti detalei mashin metodom Pro s uchetom vzaimosvyazi meghdu ego parametrami [Determination of the endurance limit of machine parts by the Pro method, taking into account the relationship between its parameters] // Detalimashin: Respondenciya meghvedomstvennogo nauchno-tehnicheskogo sbornika (Machine details: Response to an interdepartmental scientific and technical digest). 1988. - Rel. 40. - 102-104l.

5. Oleinik N.V.,Konoplev A.V.,Kibakov A.G. Metodi uskorennogo opredeleniya harakteristik soprotivleniya ustalosti v prakticheskih prilogheniyah [Methods of accelerated determination of fatigue resistance characteristics in practical applications]. Odessa: Astroprint, 2000. - 138 l.

Стаття надійшла 15.03.2021

Посилання на статтю: Коноплёв А.В., Шумило А.Н., Рожко Е.К., Галевский В.В., Кононова О.Н. Сравнительная оценка экспериментальных и расчётных значений разрушающих напряжений при испытаниях с линейно возрастающей нагрузкой // Вісник Одеського національного морського університету: 3б. наук. праць, 2021. № 2(65). C. 128-137. DOI 10.47049/ 22261893-2021-2-128-137.

Article received 15.03.2021

Reference a JournalArtic: Konoplev A.V., Shumilo A.N., Rozhko E.K., Halevskyi V.V., Kononova O.N. Comparative assessment of experimental and calculated values of breaking tensions during tests with a linearly increasing load // Herald of the Odessa national maritime university, 2021. 2(65), 128-137. DOI 10.47049/ 2226-1893-2021-2-128-137. 